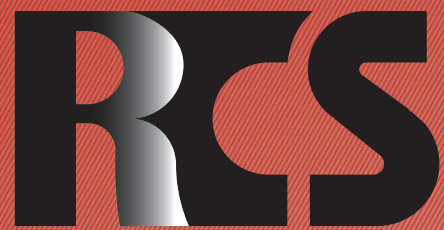

Depósito legal ppi $201502 Z U 4662$

Esta publicación científica en formato digital es continuidad de la revista impresa Depósito Legal: pp $197402 Z$ Z789

- ISSN: 1315-9518 • ISSN-E: 2477-9431

Universidad del Zulia. Revista de la Facultad de Ciencias Económicas y Sociales Vol. XXVI. No. 1

Enero-Marzo, 2021 


\title{
Formas de entender la desigualdad en la actualidad: Un nuevo camino
}

\author{
Martínez Navarro, Diego*
}

\begin{abstract}
Existe una gran controversia alrededor de la cuestión de la desigualdad desde la perspectiva puramente económica, puesto que, si bien es reconocido que la desigualdad económica tiene efectos perjudiciales sobre la sociedad (discriminación, aumento de la vulnerabilidad de las clases más desfavorecidas, descontento generalizado, explosividad social, entre otros), desde el enfoque económico existen controversia sobre si la desigualdad es totalmente prescindible o por el contrario sería deseable mantener un nivel determinado de desigualdad económica con el objetivo de maximizar la eficiencia y productividad de la economía. Asimismo, también existe un gran debate sobre cuál es la dinámica de la desigualdad a largo plazo, qué patrones se pueden encontrar y sobre qué variables se asienta.

La mayor aportación realizada en éste campo vino de la mano de Kuznets (1955) en la que establece que las economías tradicionales (mayormente agrícolas) serían equitativas, y esta equidad se rompería cuando comenzasen las migraciones del campo a las ciudades, es decir, la industrialización y también por la
\end{abstract}

concentración de los ahorros de empresarios. Estos dos factores generarían desigualdad en la economía hasta que esta se desarrollase lo suficiente como para que la acción del Estado (políticas redistributivas), la demografía (tasas de natalidad más bajas entre los más ricos y más altas entre los más pobres), el cambio tecnológico (que disminuiría el rendimiento de los activos de la vieja tecnología) y el desarrollo del sector servicios (sector con altas remuneraciones y que podría albergar a trabajadores de cualquier estrato social), harían que la economía volviese a tender hacia una situación más equitativa.

Esta teoría se ha discutido por muchos autores como Fields (1989); Greenwood y Jovanovic (1990); Deininger y Squire (1996; 1998); Higgins y Williamson (1999); Barro (2008); Rattan (2012) y MartinezNavarro, Amate-Fortes y Guarnido-Rueda (2020). Algunos aseguran que la teoría de la U-invertida de Kuznets (ver Figura I), es un fenómeno empíricamente observable mientras que otros defienden lo contrario, que es una hipótesis basada en los deseos personales de Kuznets más que en la observación. 


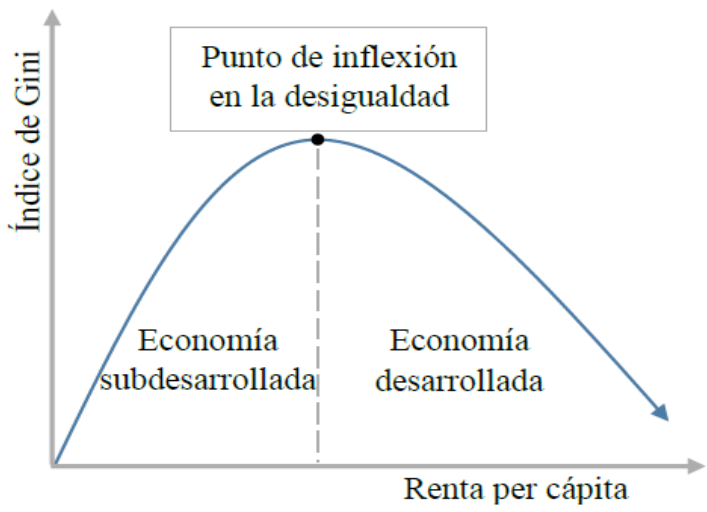

Fuente: Elaboración propia, 2020.

Figura I: Curva de Kuznets

Los economistas tuvieron que esperar hasta el 2013 para recibir otra aportación que tuviera un impacto notable en el campo (según P. Krugman se trató de "el libro más importante del año, y tal vez de la década") y que llamase lo suficiente la atención como para volver a plantear en términos precisos y firmes hacia qué dirección se encamina la desigualdad. Esta aportación fue la de Piketty (2013), en la que establece que la desigualdad tendería a aumentar siempre que la tasa de rendimiento media del capital (los beneficios, intereses, rentas, dividendos, entre otros) fuese superior que la tasa de crecimiento del PIB (ver Figura II).

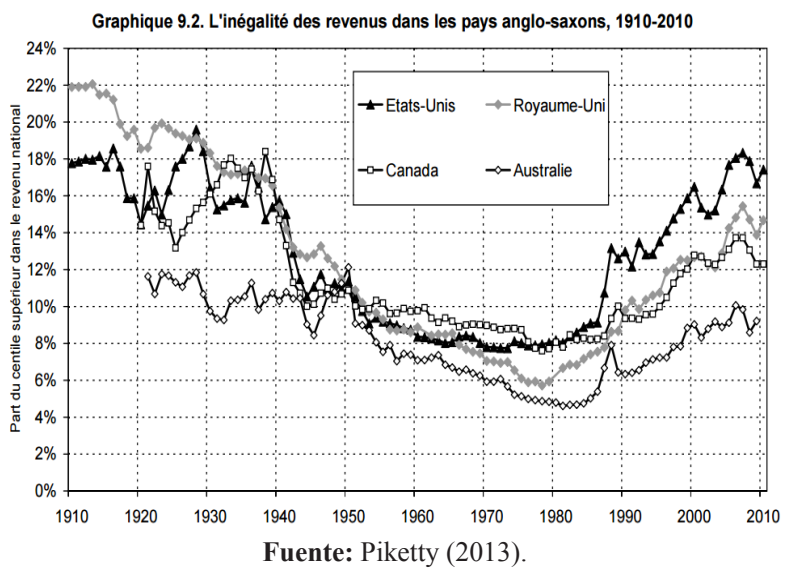

Figura II: Desigualdad de ingresos en los países anglosajones. 1970-2010 
Asimismo, el propio Piketty (2013) argumenta que la caída de la desigualdad que observó Kuznets no sólo se debía a las fuerzas naturales del mercado, sino que se correspondía a una reducción de la tasa de rendimiento del capital junto con un incremento simultáneo de la tasa de crecimiento económica. Una vez el autor francés establece estos principios, concluye que para disminuir el nivel de desigualdad es necesario imponer un impuesto sobre el capital para reducir la acumulación de renta del segmento más rico de la población y transferir esas rentas a los trabajadores.

No obstante, se pueden hallar diferencias entre los puntos de partida de Kuznets y Piketty, en primer lugar: Piketty calcula la desigualdad económica a través de la declaración de los contribuyentes mientras que Kuznets lo hacía sobre las rentas. En segundo lugar: Kuznets empleaba el PIB per cápita como medida de desarrollo mientras que Piketty empleó la tasa de crecimiento del PIB, una medida de crecimiento más que de desarrollo. Y finalmente, Kuznets se centró en el caso de Estados Unidos en el siglo XX, mientras que Piketty tuvo en cuenta a Francia, Gran Bretaña, Alemania, Suecia, Estados Unidos y Japón desde mediados del siglo XX hasta sus años más recientes.

Aquí surgió una gran cuestión, y es: Según el estudio de Kuznets (1955), y muchos otros autores que lo secundaron como Greenwood y Jovanovic (1990); Higgins y Williamson (1999); Barro (2008) y Martínez, et al. (2020), sí que se observa que la desigualdad finalmente tiene una tendencia decreciente. No obstante, por otro lado, Piketty (2013) asegura que la desigualdad tenderá a aumentar ¿qué teoría tiene más validez?

A este respecto, irrumpió en éste campo de estudio Milanovic en 2016 en la que planteó que la observación de Kuznets fue una onda de lo que podrían denominarse "las olas Kuznets", es decir, Milanovic (2016) defiende que el comportamiento de la desigualdad realmente no es ni una U-invertida como atestiguó Kuznets ni va a tener una tendencia inexpugnablemente creciente como aseguró Piketty, sino que tiene un comportamiento similar a una función seno en la que dada una cantidad de años impredecible ocurrirá un cambio económico-social que hará que el comportamiento de la desigualdad cambie su tendencia. A éste respecto, Kuznets observó un tramo creciente-decreciente y Piketty observó el tramo creciente. Mostrando Milanovic lo que, por su forma gráfica se ha denominado, la "trompa de elefante" (ver Figura III), donde se arecia la curva de Kuznets que ha sido sucedida por un incremento de la desigualdad en la última década.

$1993-2013$

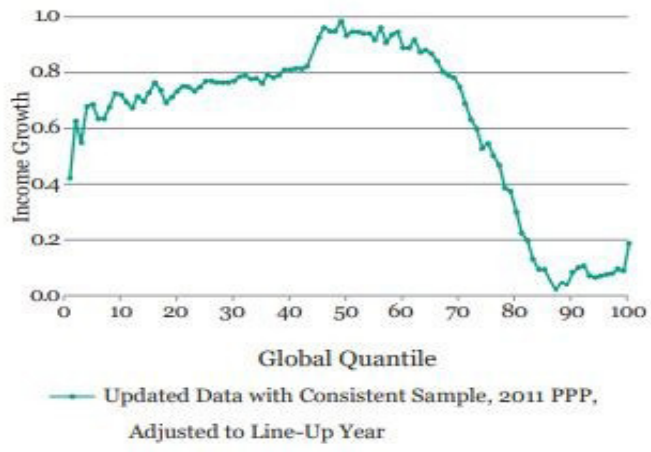

Fuente: Milanovic (2016).

Figura III: Desigualdad de ingresos a nivel mundial. 1993-2013 
Llegado a este punto, la cuestión podría plantearse del siguiente modo: Si se mantuviése las premisas de Kuznets (emplear el PIB per cápita en lugar de la tasa de crecimiento del PIB, y la desigualdad calculada a través de las rentas en lugar de a través de las declaraciones de los contribuyentes) ¿se podría verificar el elefante de Milanovic observando un patrón en forma de sinusoide? Dicho de otro modo, si se cuenta con los datos desde donde estén disponibles hasta la actualidad ¿se encontraría un patrón de U-invertida o un sinusoide?

Para responder a esta pregunta se puede recurrir a la información sobre desigualdad que ofrece la World Income Inequality Database (WIID) publicada por Solt (2020), gracias a su última actualización realizada en 2020 cubre datos de mediados del siglo XX hasta el año 2018 en más de 200 países. Esta base de datos sobre desigualdad, está confeccionada con la intención de ofrecer datos homogéneos para ayudar a realizar comparaciones tanto de horizonte transversal como temporal, así como ambas simultáneamente, justo lo necesario para este tipo de estudios, con el fin de poder realizar el contraste sobre el comportamiento de la desigualdad tanto desde una perspectiva gráfica como un análisis más exhaustivo desde el punto de vista econométrico.

Finalmente, la curva de Kuznets sobre la desigualdad continúa siendo hoy día objeto de estudio como puede verse en recientes publicaciones como Canh, et al. (2020); Le, et al. (2020); Martínez-Navarro, et al. (2020); o Topuz y Dağdemir (2020); en estas se fundamenta que se observa la curva de Kuznets basándose en análisis empíricos, lo que implicaría que la desigualdad está tendiendo a reducirse.

No obstante, se está observando un aumento generalizado de la desigualdad en el mundo, tal y como evidencian Saez y Zucman (2020) o Díez-Minguela, et al. (2020). Por lo que parece necesario que se pase a estudiar una relación cúbica entre la desigualdad y el desarrollo, en lugar de cuadrada, de manera que se contraste si es observable la trompa de elefante de Milanovic empleando análisis econométricos robustos con todos los datos disponibles como hizo Piketty y todos aquellos que en la actualidad estudian la teoría de Kuznets, y no solamente enfocarse en el período comprendido entre 1993 y 2013 como se muestra en el gráfico anterior de Milanovic (2016).

\section{Referencias bibliográficas}

Barro, R. (2008). Inequality and growth revisited. Working Paper Series on Regional Economic Integration No. 11. Asian Development Bank.

Canh, N. P., Schinckus, C., Thanh, S. D., y Hui, F. C. (2020). Effects of the internet, mobile, and land phones on income inequality and The Kuznets curve: Cross country analysis. Telecommunications Policy, 44(10), 102041. https://doi. org/10.1016/j.telpol.2020.102041

Deininger, K., y Squire, L. (1996). A new data set measuring income inequality. The World Bank Economic Review, 10(3), 565-591. https://doi.org/10.1093/ wber/10.3.565

Deininger, K., y Squire, L. (1998). New ways of looking at old issues: Inequality and growth. Journal of Development Economics, 57(2), 259-287.

Díez $\square$ Minguela, A., González $\square$ Val, R., Martinez $\square$ Galarraga, J., Sanchis, M. T., y Tirado, D. A. (2020). The long $\square$ term relationship between economic development and regional inequality: South $\square$ West Europe, 1860-2010. EHES Working Papers in Economic History, (119), 1-41.

Fields, G. S. (1989). Changes in poverty and inequality in developing countries. The World Bank Research Observer, 4(2), 167-185. https://doi.org/10.1093/ wbro/4.2.167

Greenwood, J., y Jovanovic, B. (1990). 
Financial development, growth, and the distribution of income. Journal of Political Economy, 98(5, Part 1), 1076-1107.

Higgins, M., y Williamson, J. (1999). Explaining inequality the world round: Cohort size, Kuznets curves. and openness. FRB of $\mathrm{New}$ York Staff Report, (79). http://dx.doi. org/10.2139/ssrn. 173408

Kuznets, S., (1955). Economic growth and income inequality. The American Economic Review, 45(1), 1-28.

Le, T-H., Nguyen, C., Su, T. D., y Tran-Nam, B. (2020). The Kuznets curve for export diversification and income inequality: Evidence from a global sample. Economic Analysis and Policy, 65(C), 21-39. https://doi. org/10.1016/j.eap.2019.11.004

Martínez-Navarro, D., Amate-Fortes, I., y Guarnido-Rueda, A. (2020). Inequality and development: Is the Kuznets curve in effect today? Economia Politica, 37(3), 703-735. https://doi. org/10.1007/s40888-020-00190-9

Milanovic, B. (2016). Introducing Kuznets waves: How income inequality waxes and wanes over the very long run. Voxeu.
Piketty, T. (2013). Capital in the Twentyfirst Century. The Belknap Press of Harvard University Press.

Rattan, D. (2012). Exploring the Kuznets Curve and the 'Latin American Effect' in Latin America. International Economic Review: Post Recession Challenges \& Analyses. Matrix Publishers, pp. 277-287.

Saez, E., y Zucman, G. (2020). The rise of income and wealth inequality in America: Evidence from distributional macroeconomic accounts. Journal of Economic Perspectives, 34(4), 3-26.

Solt, F. (2020). Measuring income inequality across countries and over time: the standardized world income inequality database. Social Science Quarterly, 101(3), 1183-1199. https:// doi.org/10.1111/ssqu. 12795

Topuz, S. G., y Dağdemir, Ö. (2020). Analysis of the relationship between trade openness, structural change, and income inequality under Kuznets curve hypothesis: The case of Turkey. The Journal of International Trade \& Economic Development, 29(6), 1-18. https://doi.org/10.1080/09638199.201 $\underline{9.1711146}$ 